\title{
(6) OPEN ACCESS \\ Treatment results of endosaccular coil embolization of asymptomatic unruptured intracranial aneurysms in elderly patients
}

\author{
Hidenori Oishi, ${ }^{1}$ Munetaka Yamamoto, ${ }^{2}$ Senshu Nonaka, ${ }^{3}$ Takashi Shimizu, ${ }^{4}$ \\ Kensaku Yoshida, ${ }^{5}$ Takashi Mitsuhashi, ${ }^{6}$ Hajime Arai $^{2}$
}

${ }^{1}$ Department of Neuroendovascular Therapy, Juntendo University School of Medicine, Tokyo, Japan

${ }^{2}$ Department of Neurosurgery, Juntendo University School of Medicine, Tokyo, Japan

${ }^{3}$ Department of Neurosurgery, Juntendo Urayasu Hospital,

Tokyo, Japan

${ }^{4}$ Department of Neurosurgery, Juntendo Nerima Hospital, Tokyo, Japan

${ }^{5}$ Department of Neurosurgery, Tokyo Metropolitan Hiroo Hospital, Tokyo, Japan ${ }^{6}$ Department of Neurosurgery, Tama-Nanbu Chiiki Hospital, Tokyo, Japan

Correspondence to Dr H Oishi, Department of Neurosurgery, Juntendo University School of Medicine, 2-1-1 Hongo, Bunkyo-ku, Tokyo 113-8421, Japan; ohishi@juntendo.ac.jp

Received 29 May 2014 Revised 1 July 2014 Accepted 2 July 2014 Published Online First 17 July 2014

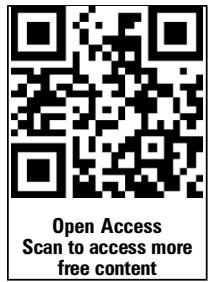

\section{CrossMark}

To cite: Oishi $\mathrm{H}$

Yamamoto M, Nonaka S,

et al. J Neurolntervent Surg 2015;7:660-665.
ABSTRACT

Background and purpose We report the results of endosaccular coil embolization of asymptomatic unruptured intracranial aneurysms (UIAs) in elderly patients ( $\geq 65$ years).

Materials There were 375 elderly patients with 400 asymptomatic UIAs. Patients were divided into two groups: group A included patients aged 65-74 years and group $B$ patients aged $\geq 75$ years.

Results Endovascular procedures were completed in $97.8 \%$ of patients. Immediate anatomical outcomes showed complete occlusion in $53.7 \%$, residual neck in $18.9 \%$, and residual aneurysm in $27.4 \%$. Anatomical follow-ups (mean 36.3 \pm 28.1 months) were unchanged in $55.7 \%$, improved in $22.6 \%$, minor recurrence in $11.5 \%$, and major recurrence in $10.2 \%$. Procedure related complications occurred in 31 patients (8.3\%). 15 patients had ischemic and 10 hemorrhagic complications. Retreatments were performed in 16 patients (4.3\%). Among 366 patients technically completed, 363 (99.2\%) showed a favorable clinical outcome (modified Rankin Scale (mRS) score $<1$ ) at 30 days. The remaining three patients showed a worsening of $\mathrm{mRS}>0$ at 30 days. The latest clinical follow-up outcomes showed: mRS score 0 in 362 patients; $\mathrm{mRS}$ score 1 in one; mRS score 3 in one; $\mathrm{mRS}$ score 4 in one; and mRS score 5 in one patient. Permanent morbidity and mortality rates were $1.1 \%$ and $0 \%$, respectively. There were no statistically significant differences in gender, comorbidities, aneurysm size, neck width, dome-to-neck ratio, immediate anatomical outcomes, anatomical follow-up outcomes, or procedure related complications between the groups.

Conclusions Endosaccular coil embolization is safe and feasible for elderly patients with asymptomatic UIAs. Old age itself should not be a contraindication.

\section{INTRODUCTION}

Asymptomatic unruptured intracranial aneurysms (UIAs) in elderly patients are increasingly diagnosed due to the development and penetration of less invasive and high quality neuroimaging modalities, such as CT angiography and MR angiography. UIAs, particularly those that are asymptomatic, have a low risk of aneurysmal subarachnoid hemorrhage (SAH). Therefore, the decision to manage elderly patients with asymptomatic UIAs requires a rigorous assessment of the risks of various treatment options and knowledge of the natural course of the disease process. Current evidence does not conclusively justify conservative management. However, the development of endovascular devices and increased experience has significantly reduced the procedure related risks of endovascular therapy of intracranial aneurysms. To our knowledge, the published series on endovascular therapy of UIAs in elderly patients is relatively small. ${ }^{1-5}$ Therefore, precise risk evaluations of endovascular therapy of asymptomatic UIAs in elderly patients have not yet been established. The purpose of this study was to report the treatment results of endosaccular coil embolization of asymptomatic UIAs in elderly patients and to elucidate the procedural feasibility, risks, and clinical outcomes.

\section{MATERIALS AND METHODS}

From April 2001 through March 2013, 1011 patients with 1087 asymptomatic UIAs underwent endosaccular coil embolization at the Juntendo University Hospital and affiliated hospitals. Patients aged 65 years or older comprised the subjects of the present study. Patients with symptomatic UIAs, dissecting or fusiform aneurysms, aneurysms treated with parent artery sacrifice, aneurysms associated with brain arteriovenous malformations, and mycotic aneurysms were excluded. Pre and post treatment clinical conditions were assessed according to the modified Rankin Scale (mRS). ${ }^{6}$ Asymptomatic UIAs associated with ruptured aneurysms were included if patients underwent curative treatment of ruptured aneurysms and could be assessed (mRS <1) at least 3 months after the onset of SAH without the influence of the SAH and/or the treatment of the ruptured aneurysm. For comparative purposes, patients were divided into two groups: group A included patients aged 65-74 years and group B patients aged 75 years or older.

The principal investigator (HO), with the opinions of the coauthors, assessed the procedure related complications, immediate/followed-up clinical outcomes, and anatomical outcomes according to the patients' medical records, outpatient charts, and surgical records. Procedure related complications were defined as neurologic deterioration, abnormal neuroimaging findings, and any treatment related undesirable events. Morbidity and mortality were defined as a deterioration of $>0$ on the $\mathrm{mRS}$ and any deaths related to the treatments. Clinical outcome and neurologic status were evaluated in detail during every outpatient visit. The coauthors reviewed all of the data. Unfortunately, 
we could not collect or analyze data of asymptomatic UIAs surgically or conservatively treated during the study period for various reasons.

The degree of aneurysm occlusion with catheter angiography was defined using the criteria previously described by Roy et al..$^{7}$ If there was no contrast filling of the dome, body, or neck of the aneurysm, it was defined as complete occlusion (CO). If the contrast medium entered part of the neck but did not fill the body and/or dome of the aneurysm, it was defined as residual neck (RN). If the contrast medium entered the body and/or dome of the aneurysm, it was defined as residual aneurysm (RA). Failure of endosaccular coil embolization was defined as impossible to place the coil or navigate the microcatheter into the aneurysm because of technical difficulties.

Our radiological follow-up protocol was MR angiography at 6 months and catheter angiography at 1 and 2 years after the procedure. After 2012, 2 year catheter angiography replaced MR angiography. If aneurysm occlusion was stable at the 2 year follow-up or at the 1 year follow-up (after 2012), the patient underwent MR angiography every year after that. MR angiography could replace catheter angiography if the patient's condition was determined to be unsuitable or if the patient refused repeated catheter angiography.

We defined the change in aneurysm occlusion as: unchanged, the area of aneurysm filling was stable; minor recurrence, an increased area of aneurysm filling without necessitating retreatment; major recurrence, an increased area of aneurysm filling ideally necessitating retreatment; and improved, a decreased area of aneurysm filling.

All of the endovascular procedures were performed with the patient under general anesthesia. Systemic heparinization was initiated in all patients to maintain an activated clotting time of 250-300 s throughout the procedure and was stopped at the end of the procedure for most patients. Patients were given aspirin $100 \mathrm{mg}$ as an antiplatelet medication a few hours before the procedure which was continued daily for 1-2 months after the procedure to prevent thromboembolic events due to thrombus formation at the neck of the coiled aneurysms. If a stent assisted technique was scheduled, dual antiplatelet therapy with aspirin $100 \mathrm{mg}$ plus clopidogrel $75 \mathrm{mg}$ was given at least 5 days before the procedure and was continued for at least 6 months. In most patients, aspirin was continued indefinitely until 1 year after the procedure. One or two working projections providing the best views of the aneurysm neck/body and microcatheter approach routes were decided on the basis of images generated by threedimensionally reconstructed rotational DSA. If that was not available, two-dimensional DSA in multiple projections was performed to obtain optimal working projections. After the procedure, angiograms in the frontal, lateral, and working projections were acquired to assess the degree of aneurysm occlusion and to rule out angiographically detectable thromboembolic events.

A simple technique of using a single microcatheter was done if a satisfactory aneurysm occlusion was expected. Adjunctive techniques (balloon assisted, double catheter, or stent assisted) were used for aneurysms with an unfavorable angioarchitecture. The appropriate sizes and shapes of the coils were selected according to the target aneurysm. As is our standard procedure, we inserted the coils within the aneurysm from the wall to the center as densely as possible or until another coil could no longer be inserted, without compromising the parent artery lumen, even after complete angiographic obliteration was achieved.

Several brands of bare platinum coils were used. The biologically modified coils (Matrix 2; Striker, California, USA) were used in 81 selected aneurysms treated after August 2008. In most of the procedures we used a braided microcatheter (Excelsior SL 10; Striker) with the tip manually steam shaped. Navigation of the microcatheter into the aneurysm was performed with the assistance of a 0.012 inch microguidewire (GT wire; Terumo, Tokyo, Japan). We usually used an over the wire balloon catheter (eg, HyperForm or HyperGlide; ev3, Irvine, California, USA) and a closed cell stent (Enterprise Vascular Reconstruction Device; Codman, Raynham, Massachusetts, USA) to perform the balloon assisted and stent assisted techniques, respectively.

Qualitative data are expressed as numbers and/or percentages. Mean values are presented as mean \pm SD. Statistical analysis was performed using the standard $\chi^{2}$ test with or without Yates' correction. Differences were considered significant at $\mathrm{p}<0.05$.

\section{RESULTS}

A total of 375 elderly patients with 400 asymptomatic UIAs were included in this study. Table 1 shows the characteristics of the patients and the sizes of the aneurysms.

Mean age was $71.0 \pm 4.7$ years, with a female predominance (73.3\%). Group A included 292 patients with 315 aneurysms and group B included 83 patients with 85 aneurysms. There were no statistically significant differences in gender/comorbidities between the groups, but group B had a high rate of aneurysms detected during examination of aneurysmal SAH $(p=0.042)$. The size and neck width of the aneurysms were measured by catheter angiography. There were no significant differences in aneurysm size, neck width, or dome to neck ratio between the groups.

Table 2 shows the locations of the aneurysms. Aneurysms located in the anterior circulation represented $83.3 \%$ of all aneurysms. Frequent locations were the anterior communicating artery, posterior communicating artery, and siphon of the internal carotid artery in the anterior circulation, and the basilar artery bifurcation in the posterior circulation. There were no statistically significant differences in aneurysm locations between the two groups.

Table 3 shows the embolization techniques used. Endovascular procedures were completed in 391 aneurysms $(97.8 \%)$ and failed in nine (2.2\%). The techniques used were simple in $37.0 \%$, balloon assisted in $44.3 \%$, double catheter in $9.0 \%$, and stent assisted in $7.5 \%$. There was no significant differences in the techniques used between the two groups. The reasons for the technical failures in nine aneurysms from nine patients were: unfavorable dome to neck ratio in four patients, wide neck in three patients, difficulty in preserving the branch originating from the aneurysm neck in one patient, and difficulty in navigating the microcatheter into the aneurysm due to the tortuosity of the cervical and intracranial vessels in one patient. Although there was no statistically significant difference, eight of the technical failures were in group A. Two of the nine patients who had a technical failure underwent surgical clipping of the aneurysm. The remaining seven patients were treated conservatively.

Table 4 shows the immediate and follow-up anatomical outcomes of 391 aneurysms which were technically completed. Follow-up anatomical outcomes were assessed by catheter angiography or MR angiography $>179$ days after treatment. Immediate anatomical outcomes showed CO in $53.7 \%$, residual neck in $18.9 \%$, and residual aneurysm in $27.4 \%$. There was no significant difference in the immediate anatomical outcomes between the groups but there was a trend to a the high rate of $\mathrm{CO}$ in group A and of residual aneurysm in group B. A total of 
Table 1 Characteristics of the patients and aneurysms

\begin{tabular}{|c|c|c|c|c|c|c|}
\hline & \multicolumn{2}{|l|}{ Overall } & \multicolumn{2}{|l|}{ Group A } & \multicolumn{2}{|l|}{ Group B } \\
\hline & $\mathbf{N}$ & $\%$ & $\mathbf{N}$ & $\%$ & $\mathbf{N}$ & $\%$ \\
\hline No of patients/aneurysms & $375 / 400$ & & $292 / 315$ & & $83 / 85$ & \\
\hline \multicolumn{7}{|l|}{ Age (years) } \\
\hline Range & $65-90$ & & $65-74$ & & $75-90$ & \\
\hline Mean & $71.0 \pm 4.7$ & & $69.1 \pm 2.8$ & & $77.9 \pm 3.2$ & \\
\hline Women & 275 & $(73.3)$ & 211 & $(72.3)$ & 64 & $(77.1)$ \\
\hline \multicolumn{7}{|l|}{ Aneurysm detection } \\
\hline Examinations for various reasons unrelated to intracranial aneurysms & 361 & $(90.3)$ & 290 & $(92.1)$ & 71 & (83.5) \\
\hline Association with ruptured intracranial aneurysms & 39 & $(9.8)$ & 25 & $(7.9)$ & 14 & $(16.5)$ \\
\hline \multicolumn{7}{|l|}{ Comorbidities } \\
\hline Hypertension & 140 & (37.3) & 110 & (37.7) & 30 & $(36.1)$ \\
\hline Diabetes mellitus & 19 & $(5.1)$ & 16 & $(5.5)$ & 3 & (3.6) \\
\hline Cerebrovascular stroke & 32 & $(8.5)$ & 22 & $(7.5)$ & 10 & $(12.0)$ \\
\hline Dyslipidemia & 10 & $(2.7)$ & 4 & $(1.4)$ & 6 & $(7.2)$ \\
\hline \multicolumn{7}{|l|}{ Aneurysm size } \\
\hline Mean $(\mathrm{mm})$ & $6.0 \pm 2.7$ & & $5.9 \pm 2.7$ & & $6.0 \pm 2.4$ & \\
\hline Neck width (mm) & $3.4 \pm 1.3$ & & $3.4 \pm 1.3$ & & $3.5 \pm 1.4$ & \\
\hline Dome to neck ratio & $1.8 \pm 0.6$ & & $1.8 \pm 0.6$ & & $1.8 \pm 0.7$ & \\
\hline
\end{tabular}

291 patients with 314 aneurysms underwent anatomical follow-up. The overall mean anatomical follow-up period was $36.3 \pm 28.1$ months (range 6 months to 10 years). The mean anatomical follow-ups with catheter angiography and with MR angiography were $20.9 \pm 17.0$ months and 31.6 \pm 26.7 months, respectively. Anatomical follow-ups showed unchanged in $55.7 \%$, improved in $22.6 \%$, minor recurrence in $11.5 \%$, and major recurrence in $10.2 \%$. There was no significant difference in anatomical follow-up outcomes between the groups but there was a trend to a high rate of improvement and a low rate of recurrence in group B. Group A had a significantly higher rate

Table 2 Aneurysm location

\begin{tabular}{|c|c|c|c|c|c|c|}
\hline \multirow[b]{2}{*}{ Location } & \multicolumn{2}{|c|}{ Overall } & \multicolumn{2}{|c|}{ Group A } & \multicolumn{2}{|c|}{ Group B } \\
\hline & $\mathrm{N}$ & $\%$ & $\mathrm{~N}$ & $\%$ & $\mathrm{~N}$ & $\%$ \\
\hline Anterior circulation & 333 & (83.3) & 263 & (83.5) & 70 & (82.4) \\
\hline Cavernous segment of ICA & 7 & $(1.8)$ & 5 & $(1.6)$ & 2 & (2.4) \\
\hline Siphon of ICA & 67 & (16.8) & 54 & (17.1) & 13 & $(15.3)$ \\
\hline PcomA & 77 & (19.3) & 59 & (18.7) & 18 & $(21.2)$ \\
\hline AchoA & 15 & (3.8) & 12 & (3.81) & 3 & (3.5) \\
\hline ICA bifurcation & 10 & (2.5) & 10 & (3.2) & 0 & (0) \\
\hline AcomA & 83 & (20.8) & 67 & $(21.3)$ & 16 & $(18.8)$ \\
\hline ACA & 27 & (6.8) & 18 & (5.7) & 9 & (10.6) \\
\hline MCA & 47 & (11.8) & 38 & $(12.1)$ & 9 & (10.6) \\
\hline Posterior circulation & 67 & $(16.7)$ & 52 & $(16.5)$ & 15 & (17.6) \\
\hline BA bifurcation & 38 & $(9.5)$ & 29 & $(9.2)$ & 9 & $(10.6)$ \\
\hline SCA & 14 & (3.5) & 10 & (3.2) & 4 & $(4.7)$ \\
\hline PICA & 3 & $(0.8)$ & 2 & $(0.6)$ & 1 & $(1.2)$ \\
\hline PCA & 4 & $(1.0)$ & 3 & $(1.0)$ & 1 & $(1.2)$ \\
\hline BA trunk & 3 & $(0.8)$ & 3 & (1.0) & 0 & (0) \\
\hline VA & 5 & (1.3) & 5 & (1.6) & 0 & (0) \\
\hline
\end{tabular}

$\mathrm{ACA}$, anterior cerebral artery; AchoA, anterior choroidal artery; AcomA, anterior communicating artery; BA, basilar artery; ICA, internal carotid artery; MCA, middle cerebral artery; PCA, posterior cerebral artery; PcomA, posterior communicating artery; PICA, posterior inferior cerebral artery; SCA, superior cerebral artery; VA, vertebral artery. of anatomical follow-up compared with group B $(82.2 \%$ vs $61.4 \% ; \mathrm{p}<0.01)$. There was a significant difference in anatomical follow-up modality between the groups $(p<0.05)$. Catheter angiography was more frequently used in group A than in group B (78.8\% vs $54.9 \%)$.

Procedure related complications occurred in 31 patients $(8.3 \%)$. Fifteen patients had ischemic complications: ischemic findings on MR or CT images in nine, angiographically thromboembolic findings during the procedure in three, and coil loop herniation with thrombus formation within the parent artery in three. Ten patients had hemorrhagic complications: intraprocedural aneurysmal rupture in six, post treatment aneurysmal rupture in two (on the day of the procedure and 9.1 years after the procedure), guidewire induced branch perforation in one, and intracranial hemorrhage most likely due to the dual antiplatelet therapy in one. The remaining six patients had other complications: carotid artery dissection during guiding catheter insertion in two, renal artery injury during femoral sheath insertion in two, transient cortical blindness due to the contrast medium in one, and transient oculomotor nerve palsy due to the coil mass in one. There was no significant difference in the risk of procedure related complications between the two groups.

A total of 366 patients with 391 aneurysms technically completed showed a mean clinical follow-up of 39.1 \pm 31.2 months (range 37 days to 11.5 years) after treatment. Among these, 363 patients $(99.2 \%)$ had a favorable clinical outcome $(\mathrm{mRS}<1)$ at 30 days after treatment. The remaining three patients showed worsening of $\mathrm{mRS}>0$ at 30 days. The causes of clinical worsening were acute in-stent thrombus formation leading to cerebral infarction, thromboembolic event leading to brainstem infraction, and post treatment aneurysmal rupture in one patient each. Two patients had clinical deterioration of $\mathrm{mRS}>30$ days after treatment. One patient suffered from cerebral infarction due to an in-stent thrombus formation 2 months after treatment; this patient had stopped taking dual antiplatelet drugs 1 week before the event. The other patient suffered from SAH 9.1 years after treatment; the patient refused retreatment of the aneurysm during the follow-up period. The overall latest clinical 
Table 3 Embolization techniques used

\begin{tabular}{|c|c|c|c|c|c|c|}
\hline & \multicolumn{2}{|c|}{ Overall } & \multicolumn{2}{|c|}{ Group A } & \multicolumn{2}{|c|}{ Group B } \\
\hline & $\mathrm{N}$ & $\%$ & $\mathbf{N}$ & $\%$ & $\mathbf{N}$ & $\%$ \\
\hline Simple & 148 & (37.0) & 119 & (37.8) & 29 & (34.1) \\
\hline Balloon assisted & 177 & (44.3) & 144 & $(45.7)$ & 33 & (38.8) \\
\hline Double catheter & 36 & (9.0) & 23 & (7.3) & 13 & (15.3) \\
\hline Stent assisted & 30 & (7.5) & 21 & $(6.7)$ & 9 & (10.6) \\
\hline Technical failure & 9 & (2.2) & 8 & $(2.5)$ & 1 & $(1.2)$ \\
\hline
\end{tabular}

follow-up outcomes showed an mRS score of 0 in 362 patients, $\mathrm{mRS}$ score of 1 in one, mRS score of 3 in one, mRS score of 4 in one, and $\mathrm{mRS}$ score of 5 in one. Therefore, the permanent morbidity and mortality rates of the patients technically completed were $1.1 \%$ and $0 \%$, respectively.

Retreatments were performed in 16 patients (4.3\%) with 16 aneurysms: once in 14 patients, and twice and three times in one patient each. The reason for retreatment was asymptomatic major recanalization in all 16 patients. Fifteen aneurysms were treated with repeat endosaccular coil embolization. Only one middle cerebral artery aneurysm was surgically treated because of the technical difficulty of repeat endosaccular coil embolization. The duration between the initial treatment and retreatment ranged from 7.1 to 109.5 months (mean 35.0 30.9 months). There was no morbidity or mortality related to the retreatment. Among the nine patients for whom the procedure technically failed, two patients underwent surgical clipping without any adverse events, and the remaining seven patients were conservatively treated with no aneurysmal SAH during the mean clinical follow-up period of $45.1 \pm 32.9$ months.

\section{DISCUSSION}

The elderly population has been growing every year and comprised about $20 \%$ of the Japanese population in 2013. The increase in radiological examinations and the development of less invasive neuroimaging techniques has resulted in more frequent incidental detection of asymptomatic UIAs in elderly patients. Aneurysmal SAH is still an important cause of death and disability in elderly patients. Reported permanent morbidity

Table 4 Immediate and follow-up anatomical outcomes

\begin{tabular}{|c|c|c|c|c|c|c|}
\hline & \multicolumn{2}{|c|}{ Overall } & \multicolumn{2}{|c|}{ Group A } & \multicolumn{2}{|c|}{ Group B } \\
\hline & $\mathrm{N}$ & $\%$ & $\mathrm{~N}$ & $\%$ & $\mathbf{N}$ & $\%$ \\
\hline Immediate anatomical outcome & 391 & & 307 & & 84 & \\
\hline $\mathrm{CO}$ & 210 & (53.7) & 171 & (55.7) & 39 & (46.4) \\
\hline RN & 74 & (18.9) & 60 & $(19.5)$ & 14 & (16.7) \\
\hline RA & 107 & (27.4) & 76 & (24.8) & 31 & (36.9) \\
\hline $\begin{array}{l}\text { Radiological follow-up }>179 \text { days } \\
\text { (No of aneurysms) }\end{array}$ & 314 & & 262 & & 52 & \\
\hline Unchanged & 175 & (55.7) & 147 & (56.1) & 28 & (53.8) \\
\hline Improved & 71 & (22.6) & 54 & (20.6) & 17 & (32.7) \\
\hline Minor recurrence & 36 & (11.5) & 32 & (12.2) & 4 & (7.7) \\
\hline Major recurrence & 32 & (10.2) & 29 & (11.1) & 3 & (5.8) \\
\hline $\begin{array}{l}\text { Follow-up modality >179 days (No } \\
\text { of patients) }\end{array}$ & 291 & & 240 & & 51 & \\
\hline Catheter angiography & 217 & (74.6) & 189 & (78.8) & 28 & (54.9) \\
\hline MR angiography & 74 & (25.4) & 51 & (21.2) & 23 & (45.1) \\
\hline
\end{tabular}

CO, complete occlusion; RA, residual aneurysm; RN, residual neck. and mortality rates range from $9.5 \%$ to $36 \%$ and from 4.8 to $23 \%$, respectively. ${ }^{8-13}$

Prevention of aneurysm rupture has been considered as an effective treatment but the natural course and treatment related risks of UIAs in elderly patients have not been clarified. Therefore, the appropriate management of these remains controversial. Recently, endovascular treatment of ruptured intracranial aneurysms has made remarkable progress and has been accepted as an alternative to surgical clipping with favorable clinical outcomes and low morbidity/mortality rates. ${ }^{14-17}$ In general, aggressive treatment of UIAs in elderly patients is not recommended because of their relatively short life expectancy and potential risks related to aging associated comorbidities. However, excessive psychological stress from the fear of aneurysm rupture can seriously affect a patient's physical and mental well being. Because all decisions on preventive treatment should be based on risk versus benefit rules, knowledge of the risks of endosaccular coil embolization for asymptomatic UIAs in elderly patients can help both physicians and patients in determining the management strategy.

Some authors found that patient age was a significant risk factor for aneurysmal SAH. ${ }^{12}{ }^{18-22}$ Morita $e t ~ a l^{23}$ reported on the nationwide study of the natural course of UIAs in the Japanese population. In their report, age $>70$ years was not an independent predictor of aneurysm rupture. In contrast, they found that older patients tended to have larger aneurysms than younger patients. Aneurysms $\geq 7 \mathrm{~mm}$ were found in $18.0 \%$ of patients younger than 50 years of age, in $21.4 \%$ of patients aged $50-59$ years, in $24.7 \%$ of patients aged $60-69$ years, in $32.6 \%$ of patients aged $70-79$ years, and in $39.7 \%$ of patients aged $\geq 80$ years $(p<0.001)$. Small aneurysms in elderly patients have a lower risk of rupture as well as those in non-elderly patients.

There have been several reports on the outcomes of endovascularly treated elderly patients with UIAs. Barker et $a l^{24}$ reported a comparison between the results of endovascular and surgical treatment of patients with UIAs. They found a better discharge disposition of patients treated with endovascular compared with surgical treatment in patients older than 65 years of age. Neurological complications were coded twice as frequently in clipped patients as in coiled patients. Cai et $a l^{25}$ reported 91\% excellent outcome ( $\mathrm{mRS} 0-1$ ) in their single center experience of 22 endovascularly treated elderly patients with UIAs. Gonzalez et $a l^{4}$ reported 91\% good outcome (mRS $\left.\leq 2\right)$ in 97 elderly patients with endovascularly treated UIAs. In a systemic review and meta-analysis of endovascular treatment of intracranial aneurysms in elderly patients ( $\geq 65$ years) by Sturiale et al, ${ }^{26}$ the rate of good clinical outcome at 1 year was $93 \%$, and the mortality rate at $6-12$ months was $10 \%$ overall in patients with UIAs. In our series, the rates of $\mathrm{mRS}<2$ at 30 days, and the latest follow-ups, were $99.7 \%$ and $99.2 \%$, respectively, comparable with previous reports.

With increasing experience and the development of devices, the procedural risks of endovascular therapy of intracranial aneurysms have been decreasing. Hwang et $a l^{2}$ reported the results of endovascular therapy of 96 elderly patients ( $>70$ years) with 122 UIAs. Adverse events and permanent neurological deficits were $4.1 \%$ and $0 \%$, respectively. Khosla et al ${ }^{1}$ reported on the results of endovascular therapy of 108 elderly patients (>65 years) with 122 UIAs. Overall major complications and major complications with neurologic deficits occurred in $17 \%$ and $8.2 \%$, respectively. Stiefel et $a l^{5}$ reported the results of endovascular therapy of 77 elderly patients ( $\geq 70$ years) with 99 UIAs. Permanent morbidity and mortality rates were $1 \%$ and $3 \%$, respectively. In the systemic review and meta-analysis by Sturiale et $a l,{ }^{26}$ transient neurological deficits 
and perioperative stroke occurred in $5 \%$ and $4 \%$, respectively. In the present series, the morbidity rate at 30 days was $1.6 \%$, and there was no mortality. These results are better than those of previous reports.

Important drawbacks of endosaccular coil embolization are the high risks of incomplete occlusion and recanalization which, leading to aneurysmal rupture and procedural failure, were comparable with surgical clipping. In the systemic review and meta-analysis by Sturiale et al, ${ }^{26}$ immediate and followed-up (>12 months) complete/near occlusion rates were $66 \%$ and $86 \%$, respectively. The rate of procedural failure was reported as $6.0 \%$. In our series, immediate and followed-up ( $>6$ months) complete/residual neck rates were $71 \%$ and $80.6 \%$, respectively. The rate of procedural failure was $2.3 \%$. Increasing physician experience, device development, and appropriate aneurysm selection warrant the satisfactory immediate anatomical results and reduced the rate of procedural failure. Sturiale et $a l^{26}$ also found that the risk of rupture after treatment, with a mixture of ruptured and unruptured aneurysms, was $2.0 \%$. In our series, two coiled aneurysms (0.5\%) ruptured after treatment. Therefore, endosaccular coil embolization proved to be an effective method of protecting asymptomatic UIAs from rupture.

Patients treated with endosaccular coil embolization of intracranial aneurysms should undergo long term radiological follow-up for detecting aneurysm recurrence and regrowth. ${ }^{27}$ Although catheter angiography is currently the standard modality, patients in group B underwent catheter angiography less often than those in group A because catheter angiography is more invasive compared with MR angiography for elderly patients. Development of a minimally invasive modality for evaluating aneurysm recurrence and regrowth is expected.

\section{CONCLUSIONS}

This study indicated that elderly patients with asymptomatic UIAs are potential candidates for endosaccular coil embolization, and old age itself should not be considered a contraindication. However, neurointerventionalists should choose patients carefully, and should perform the procedure anticipating a very low risk and a favorable clinical outcome.

\section{LIMITATIONS}

Because this study included retrospective data acquisition, and the authors assessed the anatomical/clinical outcomes and procedure related complications, the results could be biased. The patients in our series were not representative of the whole population of elderly patients with asymptomatic UIAs because untreated or surgically treated patients were not included in this study. There may have been case selection of patients who had favorable anatomical characteristics of UIAs for endovascular procedures, good neurological status, and good daily activity. Therefore, decisions regarding treatment indications may have had an inclusion bias. We could not collect or analyze data of asymptomatic UIAs of elderly patients surgically or conservatively treated during the study period for various reasons. Further systematic prospective studies with a more rigorous technical and follow-up strategy are warranted to help physicians when determining an appropriate decision in the management of asymptomatic UIAs in elderly patients.

Acknowledgements We thank Robert E Brandt, Founder, CEO, and CME of MedEd Japan, for editing the manuscript.

Contributors HO, the lead author, wrote and designed this report. MY, SN, TS, KY, and TM helped with the clinical procedures, manuscript preparation, and interpretation. HA contributed significantly to this report by critically reading the manuscript and providing many helpful suggestions. All of the authors read and approved this manuscript to be submitted for publication to this journal.

Competing interests None.

Ethics approval Study protocol was approved by our Institutional Ethics Committee.

Provenance and peer review Not commissioned; externally peer reviewed.

Open Access This is an Open Access article distributed in accordance with the Creative Commons Attribution Non Commercial (CC BY-NC 4.0) license, which permits others to distribute, remix, adapt, build upon this work non-commercially, and license their derivative works on different terms, provided the original work is properly cited and the use is non-commercial. See: http://creativecommons.org/ licenses/by-nc/4.0/

\section{REFERENCES}

1 Khosla A, Brinjikji W, Cloft H, et al. Age-related complications following endovascular treatment of unruptured intracranial aneurysms. AJNR Am J Neuroradiol 2012;33:953-7.

2 Hwang SK, Hwang G, Oh CW, et al. Endovascular treatment for unruptured intracranial aneurysms in elderly patients: single-center report. AJNR Am J Neuroradiol 2011;32:1087-90.

3 Brinjikji W, Rabinstein AA, Lanzino $G$, et al. Effect of age on outcomes of treatment of unruptured cerebral aneurysms: a study of the National Inpatient Sample 20012008. Stroke 2011;42:1320-4.

4 Gonzalez NR, Dusick JR, Duckwiler G, et al. Endovascular coiling of intracranial aneurysms in elderly patients: report of 205 treated aneurysms. Neurosurgery 2010;66:714-20.

5 Stiefel MF, Park MS, McDougall CG, et al. Endovascular treatment of unruptured intracranial aneurysms in the elderly: analysis of procedure related complications. J Neurointerven Surg 2010;2:11-15.

6 Rankin J. Cerebral vascular accidents in patients over the age of 60. II. Prognosis. Scottish Med J 1957;2:200-15.

7 Roy D, Milot G, Raymond J. Endovascular treatment of unruptured aneurysms. Stroke 2001;32:1998-2004.

8 Mont'alverne F, Musacchio M, Tolentino V, et al. Endovascular management for intracranial ruptured aneurysms in elderly patients: outcome and technical aspects. Neuroradiology 2005;47:446-57.

9 Johansson M, Norback O, Gal G, et al. Clinical outcome after endovascular coil embolization in elderly patients with subarachnoid hemorrhage. Neuroradiology 2004;46:385-91.

10 Lubicz B, Leclerc X, Gauvrit JY, et al. Endovascular treatment of ruptured intracranial aneurysms in elderly people. AJNR Am J Neuroradiol 2004;25:592-5.

11 Sedat J, Dib M, Lonjon M, et al. Endovascular treatment of ruptured intracranial aneurysms in patients aged 65 years and older: follow-up of 52 patients after 1 year. Stroke 2002;33:2620-5.

12 Inagawa $\mathrm{T}$, Yamamoto $\mathrm{M}$, Kamiya $\mathrm{K}$, et al. Management of elderly patients with aneurysmal subarachnoid hemorrhage. J Neurosurg 1988;69:332-9.

13 Ryttlefors M, Enblad P, Kerr RS, et al. International subarachnoid aneurysm trial of neurosurgical clipping versus endovascular coiling: subgroup analysis of 278 elderly patients. Stroke 2008;39:2720-6.

14 Molyneux A, Kerr R, Stratton I, et al. International Subarachnoid Aneurysm Trial (ISAT) of neurosurgical clipping versus endovascular coiling in 2143 patients with ruptured intracranial aneurysms: a randomised trial. Lancet 2002;360:1267-74.

15 Molyneux AJ, Kerr RS, Yu LM, et al. International subarachnoid aneurysm trial (ISAT) of neurosurgical clipping versus endovascular coiling in 2143 patients with ruptured intracranial aneurysms: a randomised comparison of effects on survival, dependency, seizures, rebleeding, subgroups, and aneurysm occlusion. Lancet 2005;366:809-17.

16 McDougall CG, Spetzler RF, Zabramski JM, et al. The Barrow Ruptured Aneurysm Trial. J Neurosurg 2012;116:135-44.

17 Oishi H, Yamamoto M, Shimizu T, et al. Endovascular therapy of 500 small asymptomatic unruptured intracranial aneurysms. AJNR Am J Neuroradiol 2012;33:958-64.

18 Juvela S, Porras M, Poussa K. Natural history of unruptured intracranial aneurysms: probability of and risk factors for aneurysm rupture. J Neurosurg 2008;108:1052-60.

19 Juvela S, Porras M, Heiskanen O. Natural history of unruptured intracranial aneurysms: a long-term follow-up study. J Neurosurg 1993;79:174-82.

20 Weir B, Disney L, Karrison T. Sizes of ruptured and unruptured aneurysms in relation to their sites and the ages of patients. J Neurosurg 2002;96:64-70.

21 Wiebers DO, Whisnant JP, Huston J III, et al. Unruptured intracranial aneurysms: natural history, clinical outcome, and risks of surgical and endovascular treatment. Lancet 2003;362:103-10.

22 de Rooij NK, Linn FH, van der Plas JA, et al. Incidence of subarachnoid haemorrhage: a systematic review with emphasis on region, age, gender and time trends. J Neurol Neurosurg Psychiatry 2007;78:1365-72.

23 Morita A, Kirino T, Hashi K, et al.; UCAS Japan Investigators. The natural course of unruptured cerebral aneurysms in a Japanese cohort. N Engl J Med 2012;366:2474-82. 
24 Barker FG II, Amin-Hanjani S, Butler WE, et al. Age-dependent differences in short-term outcome after surgical or endovascular treatment of unruptured intracranial aneurysms in the United States, 1996-2000. Neurosurgery 2004;54:18-28.

25 Cai Y, Spelle L, Wang H, et al. Endovascular treatment of intracranial aneurysms in the elderly: single-center experience in 63 consecutive patients. Neurosurgery 2005;57:1096-102.
26 Sturiale CL, Brinjikji W, Murad MH, et al. Endovascular treatment of intracranial aneurysms in elderly patients: a systematic review and meta-analysis. Stroke 2013;44:1897-902.

27 Thornton J, Debrun GM, Aletich VA, et al. Follow-up angiography of intracranial aneurysms treated with endovascular placement of Guglielmi detachable coils. Neurosurgery 2002;50:239-49. 